\title{
Impact of Lockdown on Patients with Fibromyalgia
}

\author{
Eleuterio A Sánchez Romero ${ }^{1,2 \star}$ (D), Lidia Martínez Rolando ${ }^{1,2}$ (D), Jorge Hugo Villafañe ${ }^{3}$ (D)
}

\author{
${ }^{1}$ Faculty of Sport Sciences, Universidad Europea de Madrid, Madrid, SPAIN \\ ${ }^{2}$ Faculty of Health Sciences, Universidad Europea de Canarias, Tenerife, Canary Islands, SPAIN \\ ${ }^{3}$ IRCCS Fondazione Don Carlo Gnocchi, Milan, ITALY \\ *Corresponding Author: eleuterio.sanchez@universidadeuropea.es
}

Citation: Sánchez Romero EA, Martínez Rolando L, Villafañe JH. Impact of Lockdown on Patients with Fibromyalgia. Electron J Gen Med. 2022;19(3):em366. https://doi.org/10.29333/ejgm/11798

\section{ARTICLE INFO}

Received: 26 Dec. 2021

Accepted: 12 Feb. 2022

\begin{abstract}
On March 11, 2020, WHO, faced with the levels of spread and the seriousness of the situation, declares COVID-19 a pandemic. In European countries and almost everywhere else in the world, people are confined to their homes in view of the imminent situation, producing reduced mobility to essential activities. This lockdown has had repercussions at different levels: (i) Inaccessibility to face-to-face healthcare, including the loss of multidisciplinary support programs for chronic pain patients. (ii) Physical reduction or inactivity due to overloaded childcare and housework, lack of space or online resources for practice, and/or as a consequence of unrefreshing sleep. (iii) Feelings of loneliness, loss of social support, uncertainty in the provision of health care if needed, and fear of contagion when exposed to open environments for essential activities, among others. (iv) Permanent or temporary loss of employment, implementation of teleworking or remote work, modifying the usual spaces of work occupation, and the interpersonal relationships inherent to this activity. (v) Vulnerability, especially in elderly population groups, associated with exposure to a large amount of information from reliable or unreliable sources, making it difficult to understand, and make decisions. (vi) Loss of family members and relatives without the social support inherent to these events.
\end{abstract}

The sum of all of them entails a series of consequences in the lives of citizens that do not go unnoticed. The use of telerehabilitation offers the possibility of providing an accesible therapeutic context (assessment, treatment, and follow-up) in situations that limit patient's relationship with the environment such as the confinement suffered. It is in this sense where it would be of interest to consider the application of these telematic therapeutic routes in other causes of lockdown or lack of accessibility due to physical, programmatic, social, or transport barriers, with the aim of reaching this target population by providing personalized care.

Keywords: lockdown, telemedicine, chronic pain

\section{IMPACT OF LOCKDOWN ON PATIENTS WITH FIBROMYALGIA}

Fibromyalgia (FM), is defined as a chronic syndrome characterized by diffuse musculoskeletal pain, associated with characteristic signs and symptoms such as fatigue and/or sleep and mood disorders; and whose etiology, pathogenesis and prognosis may or may not be known [1]. Since then different authors have pointed out different factors that could be involved, among them: dysfunction of the central nervous system (CNS), autonomic and immune systems, altered secretion of neurotransmitters, hormones, environmental stressors, and/or psychiatric issues. The prevalence of this syndrome worldwide stands at $2.1 \%$, being higher in women than in men with a ratio of 4:1 [2]. This high prevalence entails high socioeconomic costs both directly (medical visits, complementary tests, pharmacological, and nonpharmacological treatments) and indirectly (reduction in working hours, sick leave, and permanent disability). Therefore, the presence and interaction of biological, psychological, and social aspects have to be treated under the same paradigm.
On March 11, 2020, WHO, faced with the levels of spread and the seriousness of the situation, declares COVID-19 a pandemic. In European countries and almost everywhere else in the world, people are confined to their homes in view of the imminent situation, producing reduced mobility to essential activities.

It is for this reason that COVID-19 should also be analyzed from this perspective, as it has led to the use as a legislative tool of the imposition of social distancing and lockdown, resulting in a sudden isolation of the population that can produce negative effects on general health, including a worsening of physical, and mental health with the consequent risk of generating maladaptive strategies such as substance abuse or the emergence of suicidal ideation [3]. This last aspect has been highlighted in other studies [4,5] who state that mood alterations can increase suicidal ideation and that this is evidenced by an increase in calls to suicide prevention hotlines.

The authors in [6] state that loneliness, alienation, and lack of social support and uncertainty in the provision of health care are innate feelings of pandemic and confinement and may affect the prognosis and development of chronic pain. In this line, the authors in [4] refer to the growing evidence that stressors, regardless of the category to which they belong, 
directly and negatively affect the central sensitization process, worsening the symptoms of the syndrome. This statement is analyzed by the authors in [6] who conclude that intolerance to stress is a distinctive aspect of syndromes that present chronic pain such as FM, and that exposure to stressful and traumatic situations, such as those derived from COVID-19, increase the central sensitization of patients. Likewise, with respect to how psychological factors affect the intensity and interference of pain, it appears that high levels of catastrophizing, sleep disturbances, anxiety, depression and increased consumption of drugs, and medication are associated with increased pain and interference; whereas stress is only related to pain interference. It has also been observed that the absence of exercise or physical activity can worsen the functionality of subjects with chronic pain and that there is a bidirectional relationship between sleep disturbances and pain, since pain implies a disturbance in the quality and quantity of sleep and lack of sleep exacerbates pain.

This prolonged period marked by the absence of interpersonal relationships, isolation, fear of the disease, uncertainty in different areas, and other factors already mentioned leads to the assertion that the consequences of this pandemic, and specifically of the home confinement that took place between March and May in Europe, extend beyond the biological. It is at this point where lies the importance of understanding and approaching FM from a biopsychosocial perspective in which having an interdisciplinary health team and a support network is a priority so that the impact of traumatic and stressful situations such as the present pandemic is the least possible. The absence of consultations and the interruption of face-to-face therapy has led to the exploration of telerehablitation which consists of the development of new telematic therapeutic routes or the use of existing ones such as videocall platforms which allow an approach that accompanies the patient in the process and minimize the consequences described, favoring self-efficacy and the maintenance of a routine, paying special attention to psychosocial factors and the resulting physical inactivity.

There is currently no gold standard method with which to treat patients with FM. FM management is considered a multimodal process involving pain education, exercise therapy, and physical therapy. Conservative nonpharmacological treatment has shown to relieve symptoms in patients with early stage of FM disease or in the long term. Applying a multimodal treatment that includes telerehabilitation (relatively new therapeutic technique) can be easily applied by physicians in different clinical settings in the National Health Service worldwide [7]. Our hypothesis opens ways for future research in the modulation of pain pathways, perhaps offering targets to optimize pain management in FM. Understanding the associations between FM and lockdown can lead to improve treatment approaches, focusing on the most efficient therapies.

Therefore, it can be concluded that from a physiotherapy perspective a multimodal approach including pain education and therapeutic exercise produces improvements in pain, fatigue, mental health and physical function and that the use of telerehabilitation offers the possibility of providing an accesible therapeutic context (assessment, treatment, and follow-up) in situations that limit patient's relationship with the environment such as the confinement suffered. It is in this sense where it would be of interest to consider the application of these telematic therapeutic routes in other causes of lockdown or lack of accessibility due to physical, programmatic, social, or transport barriers, with the aim of reaching this target population by providing personalized care.

Author contributions: All authors have sufficiently contributed to the study, and agreed with the results and conclusions.

Funding: No funding source is reported for this study.

Declaration of interest: No conflict of interest is declared by authors.

\section{REFERENCES}

1. Wolfe F, Clauw DJ, Fitzcharles M-A, et al. 2016 Revisions to the 2010/2011 fibromyalgia diagnostic criteria. Semin Arthritis Rheum. 2016;46(3):319-29. https://doi.org/10.1016 /j.semarthrit.2016.08.012 PMid:27916278

2. Mas AJ, Carmona L, Valverde M, Ribas B, EPISER Study Group. Prevalence and impact of fibromyalgia on function and quality of life in individuals from the general population: Results from a nationwide study in Spain. Clin Exp Rheumatol. 2008;26(4):519-26. PMid:18799079

3. Global Burden of Disease 2020 Health Financing Collaborator Network. Tracking development assistance for health and for COVID-19: a review of development assistance, government, out-of-pocket, and other private spending on health for 204 countries and territories, 1990-2050. Lancet. 2021 Oct 9;398(10308):1317-43. https://doi.org/10.1016/S0140-6

4. Mohabbat AB, Mohabbat NML, Wight EC. Fibromyalgia and chronic fatigue syndrome in the age of COVID-19. Mayo Clin Proc Innov Qual Outcomes. 2020;4(6):764-6. https://doi.org /10.1016/j.mayocpiqo.2020.08.002 PMid:33204998 PMCid: PMC7661943

5. Pedersini $P$, Villafañe $J H$, Corbellini $C$, Tovani-Palone MR. COVID-19 pandemic: A physiotherapy update. Electron J Gen Med. 2021;18(1):em264. https://doi.org/10.29333/ ejgm/8574

6. Serrano-Ibáñez ER, Esteve R, Ramírez-Maestre C, RuizPárraga GT, López-Martínez AE. Chronic pain in the time of COVID-19: Stress aftermath and central sensitization. $\mathrm{Br} \mathrm{J}$ Health Psychol. 2021;26(2):544-52. https://doi.org/10.1111 /bjhp.12483 PMid:33099793

7. Pedersini $P$, Corbellini $C$, Villafañe JH. Italian physical therapists' response to the novel COVID-19 emergency. Phys Ther. 2020;100(7):1049-51. https://doi.org/10.1093/ ptj/pzaa060 PMid:32280973 PMCid:PMC7184495 